\title{
Imaging after laparoscopic sleeve gastrectomy - literature review with practical recommendations
}

\author{
Małgorzata Deręgowska-Cylke ${ }^{1 A, B, D, E, F}$, Piotr Palczewski ${ }^{1 A, B, D, E, F}$, Radosław Cylke ${ }^{2 B, D}$, Paweł Ziemiański ${ }^{2 B, D}$, \\ Wojciech Lisik ${ }^{2 D, E}$, Marek Gołębiowski ${ }^{1 A, D, E}$
}

${ }^{1} 17^{\text {st }}$ Department of Clinical Radiology, Medical University of Warsaw, Warsaw, Poland

${ }^{2}$ Department of General and Transplant Surgery, Medical University of Warsaw, Warsaw, Poland

\begin{abstract}
In the XXI century obesity has become one of the most demanding epidemiological threats worldwide. At the same time, bariatric surgery has established itself as an effective treatment for morbidly obese patients, with laparoscopic sleeve gastrectomy (LSG) emerging as the most popular bariatric procedure. This paper reviews the role of imaging studies of patients after LSG. Computed tomography is widely considered as the method of choice in detection of complications in early postoperative period. The dynamic character of upper gastrointestinal examination allows for the assessment of passage through the gastric remnant. The paper also discusses evaluation of the shape and volume of the gastric remnant assessed by imaging studies.
\end{abstract}

Key words: laparoscopic sleeve gastrectomy, complications, upper gastrointestinal series, computed tomography.

\section{Introduction}

Progress in the field of biology and medicine in the XX and XXI century has shifted the epidemiological threats, with civilizational diseases, such as obesity, being recognized as one of the most urgent health challenges to the global population [1,2]. It is estimated that there will be up to $38.1 \%$ overweight and $19.7 \%$ obese adults by 2030 worldwide, while these numbers in the United States of America (USA) alone will equal $86.3 \%$ and $51.1 \%$, respectively $[3,4]$. In Poland, overweight and obesity is also an important health, social, and economic issue. The World Health Organization (WHO) expects the prevalence of obesity in Poland to reach $28 \%$ among men and 18\% among women by 2030 [5]. Particularly worrying is the rapidly increasing prevalence of overweight and obesity in young people [6,7].

The role of surgery in the treatment of obesity has been well established over the years, and it is now not only considered the only method resulting in long-lasting significant weight loss effect, but also it has been proven to be more effective than medical therapy in the treatment of comorbidities of obesity [8-10]. The Roux-en-Y gastric bypass (RYGB) and laparoscopic sleeve gastrectomy (LSG) are the most commonly performed surgical bariatric procedures, mainly because of their high success rates and relatively low complication rates. In recent years, the number of LSGs performed worldwide has shown the highest increase rate among other metabolic surgeries [11]. According both to the International Federation for the Surgery of Obesity (IFSO) and the American Society for Metabolic and Bariatric Surgery (ASMBS), LSG has become the most popular bariatric procedure nowadays $[12,13]$. LSG is perceived as one of the safest bariatric operations. The main advantages of LSG are as follows: a relatively simple surgical technique with no need of anastomosis creation, short learning curve, and low rate of metabolic complications [14].

Correspondence address:

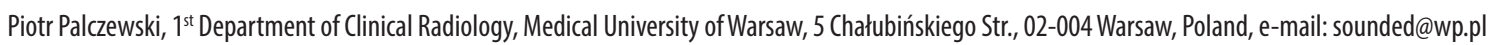

Authors' contribution:

A Study design · B Data collection · C Statistical analysis · D Data interpretation · E Manuscript preparation · F Literature search · G Funds collection 
Because a growing number of surgery clinics are decideing to start a bariatric program, it is highly possible that radiologists, working both in hospital and outpatient clinic environments, will be faced with interpretation of the images of patients after LSG. With the modifications in peri- and postoperative care protocols, the approach to the imaging algorithm is also changing. The purpose of the study is to review available literature and guidelines for imaging after LSG. We will present a surgical technique of LSG performed in our hospital and discuss the methodology and diagnostic capabilities of the main imaging methods - upper gastrointestinal (UGI) series and computed tomography (CT) - with an emphasis on the detection of postoperative complications. Currently, the main method of imaging complications after LSG is CT. UGI is performed less frequently. It can be conducted when leakage or stenosis of the sleeve is suspected or for medical research purposes (to examine the anatomy, shape, or volume of the gastric remnant and its correlation with clinical data) $[15,16]$. The other methods are auxiliary.

\section{Surgical technique}

In our hospital, LSG is the most commonly performed bariatric procedure. The first LSG in our hospital was performed in 2011, and since then 50 to 80 operations have been performed annually. According to the "Polish recommendations for bariatric and metabolic surgery" document, the main indications for bariatric surgery are BMI (body-mass index) $\geq 40$ or BMI $\geq 35$ with coexistence of obesity-specific comorbidities [17]. Each patient

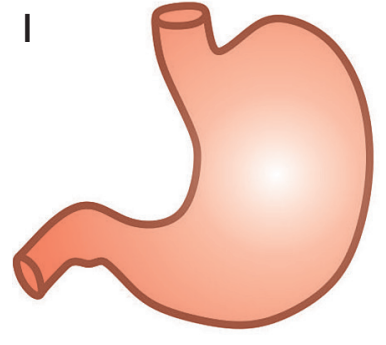

III
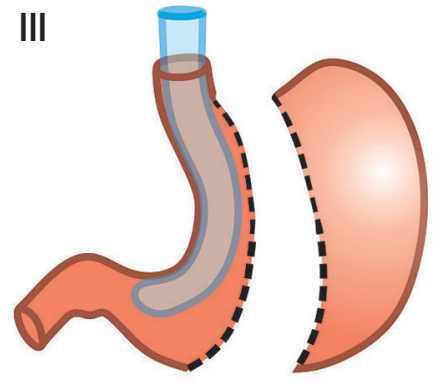

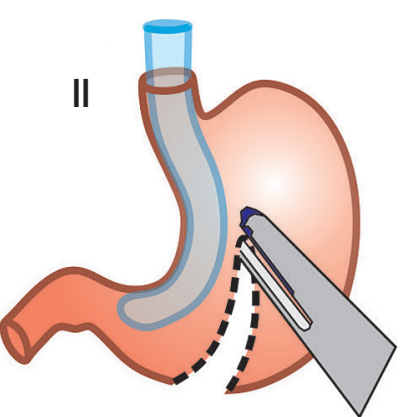

IV

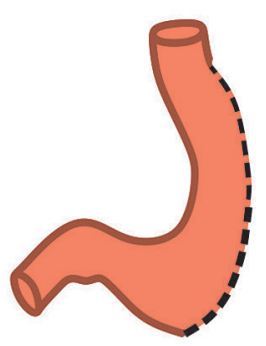

Figure 1. Schematic diagram of laparoscopic sleeve gastrectomy performance is meticulously prepared before final qualification undergoing surgical, cardiological, and anaesthesiological consultations, as well as gastroscopy. Women are also referred for the gynaecological examination. LSG is a restrictive type of bariatric surgery. The operation is performed laparoscopically by 3 surgeons under general anaesthesia. Typically, 5 trocars are used in order to achieve the best exposition. Dissection of the greater curvature of the stomach is performed using a specialist energy device. The width of the sleeve is calibrated using a 36 Fr nasogastric tube, and the resection of the stomach is performed using a linear cutting stapler only (Figure 1). At the beginning of the bariatric program, the staple line was oversewed; however, this step was given up when evidence showed that it does not reduce the risk of leakage [18]. Routinely, UGI was performed on the first day after the operation to rule out leakage, but this approach was abandoned with the end of 2017, when the Early Recovery After Bariatric Surgery (ERABS) protocol was incorporated (Table 1). Along with the introduction of this protocol, early mobilisation of the patients was possible, which shortened the length of hospital stay. It now does not exceed 3 days for patients with uncomplicated postoperative course.

\section{Imaging following laparoscopic sleeve gastrectomy}

\section{Methodology}

\section{Upper gastrointestinal}

UGI series is the most basic study after LSG. This minimally invasive technique has a long history of being used for the detection of both early and late postoperative complications $[19,20]$. The examination begins with a single abdominal radiograph in erect position to detect free gas in the peritoneum (which can be noticeable up to 1 week after surgery) and to check the position of drains [21,22]. Then, the patient swallows water-soluble contrast medium [23]. The passage through the upper GI is observed by fluoroscopy, and single X-rays are taken in anterior-posterior (AP), oblique, and lateral projections [24]. Because $\mathrm{X}$-rays of the patient are taken in both vertical and supine positions, care must be taken to ensure that the examination table can withstand the weight of a particularly obese patient [25]. In our Department of Radiology, the UGI study begins with a single abdominal X-ray in standing position (AP projection). Then, the patient is given a glass of $50 \mathrm{~mL}$ water-soluble contrast medium to swallow, and the passage of the contrast is observed using fluoroscopy we examine closely the gastroesophageal junction, sleeve morphology, the staple line, and the passage of contrast medium to the duodenum and further parts of small intestine. Afterwards, the stomach remnant is observed in oblique and lateral projections while the patient is still standing. Next, the patient takes another sip of the con- 
Table 1. Enhanced recovery after bariatric surgery (ERABS) protocol

\begin{tabular}{|l|l|l|}
\hline Preoperative & Intraoperative & Postoperative \\
\hline Pre-admission counselling & Minimally invasive surgical approach & No urinary catheters (or early removal) \\
\hline Carbohydrate loading & Local anaesthetic & No naso-gastric tubes \\
\hline No premedication & Short incisions & Early mobilisation \\
\hline \multirow{2}{*}{} & Avoidance of sodium/fluid overload & Non-opiate oral analgesic \\
\cline { 2 - 3 } & Short acting anaesthetic agents & Prevention of nausea and vomiting \\
\cline { 2 - 3 } & No drains & Early oral nutrition \\
\hline
\end{tabular}

trast medium and is told to lie on their back and on both sides on a bed. After a few minutes, additional X-rays are performed to make sure that there are no signs of leakage. Overhead radiographs following initial fluoroscopy are strongly recommended in this procedure.

\section{Computed tomography}

In recent years, CT has been increasingly used as a primary postoperative examination after bariatric procedures for complication detection. The main concern associated with the use of CT is its high radiation dose; however, it has been shown that with a proper protocol an average effective dose of $7.8 \mathrm{mSv}$ is achievable in bariatric patients, which is not much higher than for a UGI study (approximately $6 \mathrm{mSv}$ according to the American College of Radiology) [26].

CT scans from the distal part of the oesophagus to the pelvis are recognized as a default range for patients after LSG to rule out possible complications [21]. The CT protocol depends on the clinical situation. The study always begins with an unenhanced phase. If there is a clinical suspicion of active bleeding or unenhanced phase shows acute haematoma (a fluid collection with attenuation values 40-60 HU), in our opinion it is better to administer intravenous contrast before oral contrast in order to avoid the influence of possible beam hardening artefacts on the interpretation of images. To identify the source of bleeding, arterial phase CT angiographic images are acquired with bolus tracking technique. In other cases, where leak is a primary concern, the patient is required to drink a cup of oral contrast medium on the table (approximately $60 \mathrm{ml}$ ). Afterwards, intravenous contrast is administered and portal venous phase images are acquired after 70 s. Delayed phase images were suggested for exclusion of strictures or delayed passage; however, we do not use those routinely because we consider a UGI study to be much more informative in this respect [16].

There is no consensus regarding optimal patient preparation before the CT study. Some authors advocate administration of the solution of sodium bicarbonate and tartaric acid diluted with water or iodinated contrast agent directly before the study in order to expand the stomach remnant [27-31]. When the study is performed for evaluation of the stomach volume, such preparation increases the precision of measurements. For a better quality of the examination, some authors suggest intravenous injection of butylscopolamine prior to scanning [28,30,31].

In uncomplicated cases, CT scans show a tubular gastric sleeve with a staple line along the great curvature. In the area of excised stomach, abundant mesenteric fat as well as small residual pneumoperitoneum (in the early postoperative period) can be found [19,32-34]. No fluid collections should be present.

\section{Morphological and functional assessment}

\section{Shape variations}

Among the basic information obtainable from the UGI study is a shape characterization of the gastric remnant. Werquin et al. described 5 shape patterns of residual stomach: tubular, superior pouch, inferior pouch, superior-inferior pouch, and pseudodiverticular pattern [35] (Figure 2). The division is based on the staple line curvatures. The most common is tubular pattern. It is represented as a long, round, tube-shaped form with a homogeneous opacification after swallowing the contrast material. The superior pouch refers to a wider superior compartment of the sleeve, near the gastroesophageal junction, which gradually fills with contrast medium and usually creates an air-fluid level. In turn, the inferior pouch represents a wider component in the antral region. The superior-inferior pouch consists of dilatations in both cardiac and antral area. The pseudodiverticular pattern is a diverticular dilatation of the lesser curvature of the gastric sleeve [20,36-38]. The superior pouch pattern may imitate a gastric extraluminal effluent and can generate false positive diagnosis of a leakage [36,37]. Especially small superior pouches with irregular upper or outer contour and small pouches with narrow neck and filiform opacification may mimic leakage [37]. Thus, it is important to be aware of different gastric sleeve shape patterns. Shape variations seem to have an influence on the presence of gastroesophageal reflux syndrome. Occasional regurgitations and vomiting occurring in patients with the tubular shape can be explained by increased intragastric pressure in the upper region of the stomach [20]. On the other hand, the majority of authors report that the superior pouch shape exhibits a stronger connection with the 

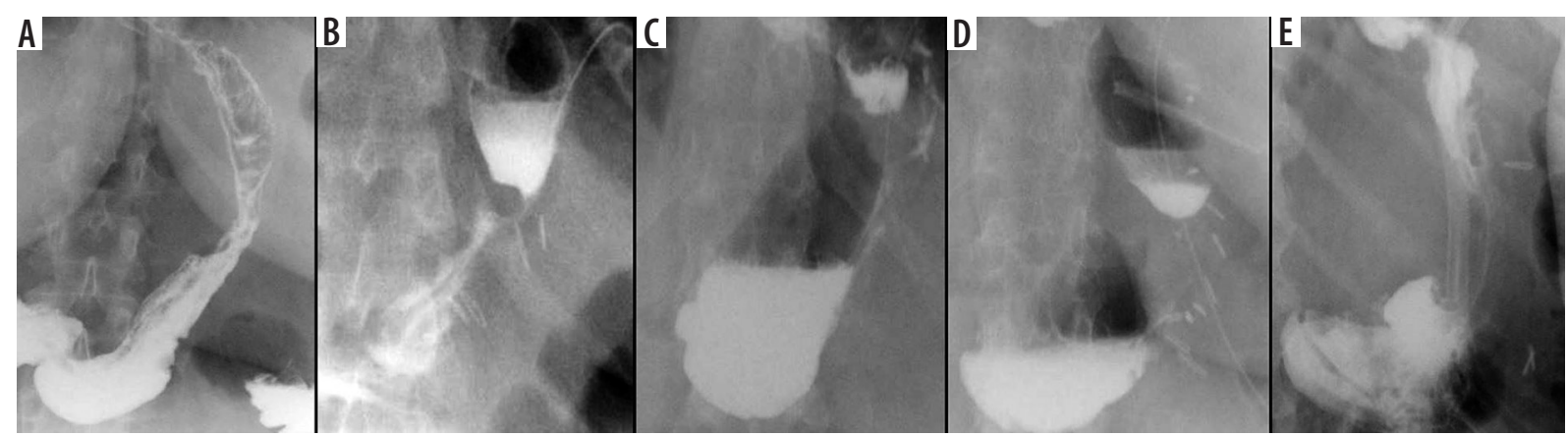

Figure 2. Shape variants of gastric remnants after laparoscopic sleeve gastrectomy (upper gastrointestinal study): A) tubular, B) superior pouch, C) inferior pouch, D) superior-inferior pouch, E) pseudodiverticular shape

gastroesophageal reflux symptoms when compared to the tubular shape $[16,37,38]$.

\section{Gastric remnant volume}

Measurement of the gastric volume after sleeve gastrectomy is not performed routinely, but it can provide valuable data. The gastric volume in the early postoperative period may relate to long-term results in weight loss reduction [39-42]. It can also provide feedback for surgeons who are at the beginning of their learning curve, helping them to create an optimal gastric sleeve. Various methods to evaluate gastric volume on UGI series were described (Table 2). One of them compares the gastric sleeve shape to an ellipsoid, and the volume can be estimated by using the mathematical formula for this figure $\left(V=4 /{ }_{3} \times \pi \times a \times\right.$ $b \times c$, where $V=$ gastric volume, $a, b, c=$ the ellipsoid's 3 different radiuses) $[42,43]$. Two views in perpendicular projections are needed in this approach. Another option is built upon the similarity of the gastric sleeve to a cylinder and uses the following formula: $V=\pi \times r^{2} \times h$, where $V=$ gastric volume, $h=$ height, and $r=$ radius $[39,40]$. The height of the cylinder is measured along the long axis of the gastric sleeve, from the cardia to the pylorus. The radius is estimated from the arithmetic mean of 3 maximum widths (measured in the upper, the medium, and the lower part of the residual stomach) divided by 2 . In an alternative approach, the volume of the stomach remnant is calculated using the sum of 2 formulas: for a cylinder and for a truncated cone $\left(V=1 /{ }_{3} \pi\left(R^{2}+r^{2}+R r\right) \times h\right.$, where $V=$ gastric volume, $R=$ maximum radius, $r=$ minimum radius, and $h=$ height) [41]. The abovementioned formulas are not highly precise because gastric sleeve shapes are not ideal geometric figures [40]. More precise measurements are possible using multidetector CT with oral contrast agent and effervescent agent, because radiological workstations enable semiautomatic volume measurements both on multiplanar and 3D displays $[28,44,45]$. The optimal gastric volume is still being debated; however, most publications suggest gastric sleeve volumes oscillating between 50 and $120 \mathrm{ml}[40,41,44,46]$.

\section{Passage}

The UGI series is the only technique allowing close evaluation of the contrast passage through the gastric remnant in a real time. Goitein et al. identified 2 patterns of contrast medium passage after LSG: a rapid, uninterrupted contrast transit through the gastric sleeve from the gastroesophageal junction through the pylorus to the duodenum (passage $<30$ seconds) and a delayed contrast flow to the duodenum (passage $>30$ seconds) $[47,48]$. Patients from the first group were found to have better fluid tolerance in the early postoperative period and were discharged earlier than the second group [47]. Prolonged contrast transit to the duodenum in the early postoperative period most often results from oedema of the pylorus wall [34]. Similarly, Pomerri et al. categorized patients into 2 groups: fast passage group - when gastric emptying takes < 1 minute; and slow passage group - when gastric emptying lasts $\geq 1$ minute [42]. They observed that the patients with fast gastric voiding had a higher weight loss 1 year after LSG with percentage of excess BMI loss $(\% \mathrm{EBL})>50 \%$ as compared to the patients with slow

Table 2. Different approaches to calculate gastric remnant volume

\begin{tabular}{|l|c|c|c|}
\hline Shape & Ellipsoid & Cylinder & Cylinder + Truncated cone \\
\hline Projection & & & \\
& & & \\
\hline Formula & $\mathrm{V}=4 / 3 \times \pi \times \mathrm{a} \times \mathrm{b} \times \mathrm{c}$ & $\mathrm{V}=\pi \times \mathrm{r}^{2} \times \mathrm{h}$ & $\mathrm{V}=\pi \times \mathrm{r}^{2} \times \mathrm{h}+1 / 3 \times \pi \times\left(\mathrm{R}_{1}{ }^{2}+\mathrm{R}_{2}{ }^{2}+\mathrm{R}_{1} \times \mathrm{R}_{2}\right) \times \mathrm{H}$ \\
\hline
\end{tabular}

$\mathrm{V}$ - volume; $\mathrm{a}, \mathrm{b}, \mathrm{c}$ - different radiuses of ellipsoid; $r$ - cylinder radius; $\mathrm{h}$ - cylinder height; $\mathrm{R}_{1}$ - minimal radius; $\mathrm{R}_{2}$ - maximal radius; $\mathrm{H}$ - height of truncated cone 
passage, who often had an inadequate weight loss (\%EBL $<50 \%)$ at all follow-up time points [42].

\section{Detection of complications}

\section{Leak}

The reported incidence of leakage after LSG ranges between 1.09 and $5.3 \%$, and it is a very dangerous complication that can lead to peritonitis, sepsis, formation of an abscess or a fistula, and even death [49-51]. Clinical manifestations of this particular complication are unspecific and include abdominal pain, fever, and tachycardia $[23,52]$. Leaks after LSG generally occur in the early postoperative period; however, they may occur up to 8 days after the surgery. According to the surgical literature, 2 types of leak are recognised: type I or subclinical (small, local, without diffusion through a fistula, and no presence of methylene blue after oral administration in any of the abdominal drains) and type II or clinical (leakage with early septic manifestation and extensive dissemination to the pleural or abdominal cavity with presence of methylene blue in any of the abdominal drains) [52-54]. Radiographically, leakage is defined as a flow of the con- trast medium through the staple line into the peritoneal cavity [55]. On UGI series leakage can present either as small effluents or more defined collections of extraluminal contrast medium $[19,56]$. However, it should be emphasised that UGI has a low leak detection rate. While most papers have reported high specificity (up to $100 \%$ in selected papers), the sensitivity is unacceptably low, ranging from $0 \%$ to $33 \%$ [57-60]. If there is a suspicion of leak, either clinically or on the UGI study, a CT scan should be performed to help with a swift and confident diagnosis establishment (Figure 3). CT has a much higher leak detection rate (up to 86\%) [49,61]. On CT, leak can present as extravasation of oral contrast medium into the peritoneum and sometimes into drains, fluid collection, or free fluid (Figure 4). When there are perigastric fluid collections, the absence of contrast medium extravasation does not preclude an active leak [62]. Nedelcu et al. created a CT classification of leak after LSG [63]. This classification recognizes 4 types of leak based on the size and location of fluid collections: type I $-<5 \mathrm{~cm}$ in the left upper quadrant of the abdomen, type II $->5 \mathrm{~cm}$ in the left upper quadrant of the abdomen with two subtypes: $\mathrm{a}$ - negative leak visualisation or $\mathrm{b}$ - positive leak visualisation, type III - diffuse abdominal collections, type IV -
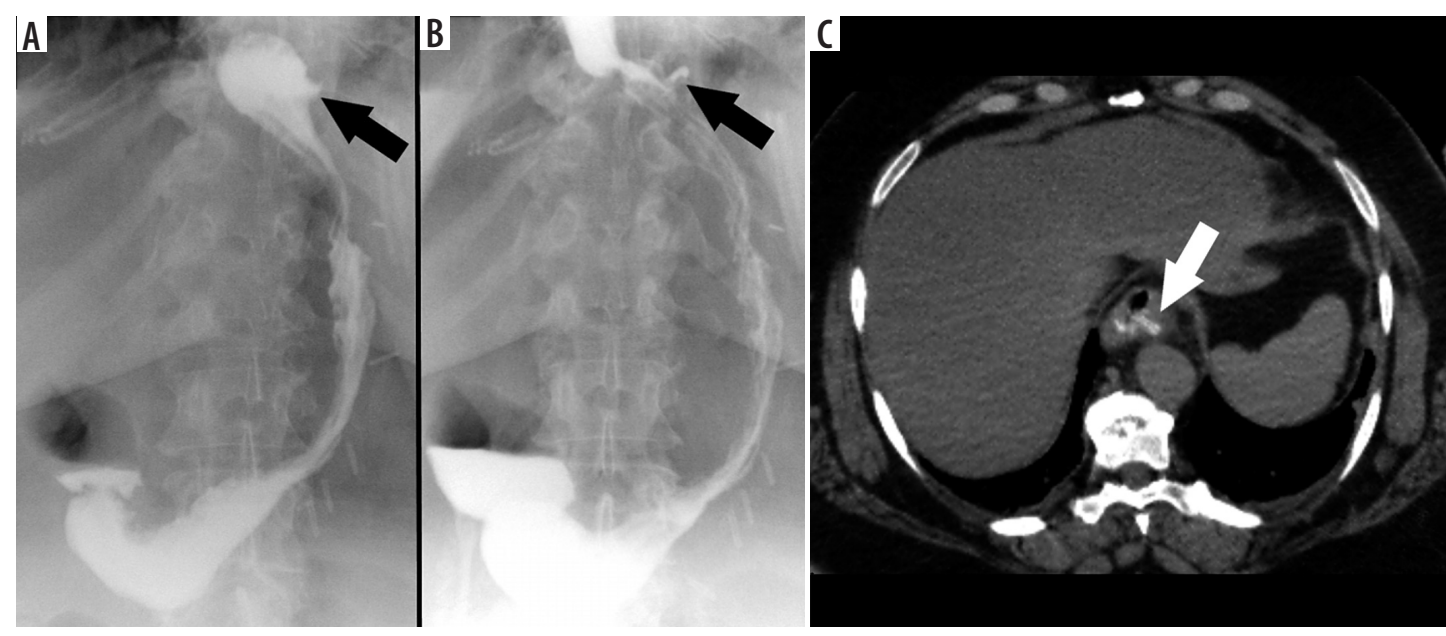

Figure 3. Example of a false positive leak result in upper gastrointestinal series. Unusual surplus of contrast medium nearby the gastroesophageal junction (black arrows on A and B). On computed tomography scan (C), there is a pseudodiverticulum in this location (white arrow)
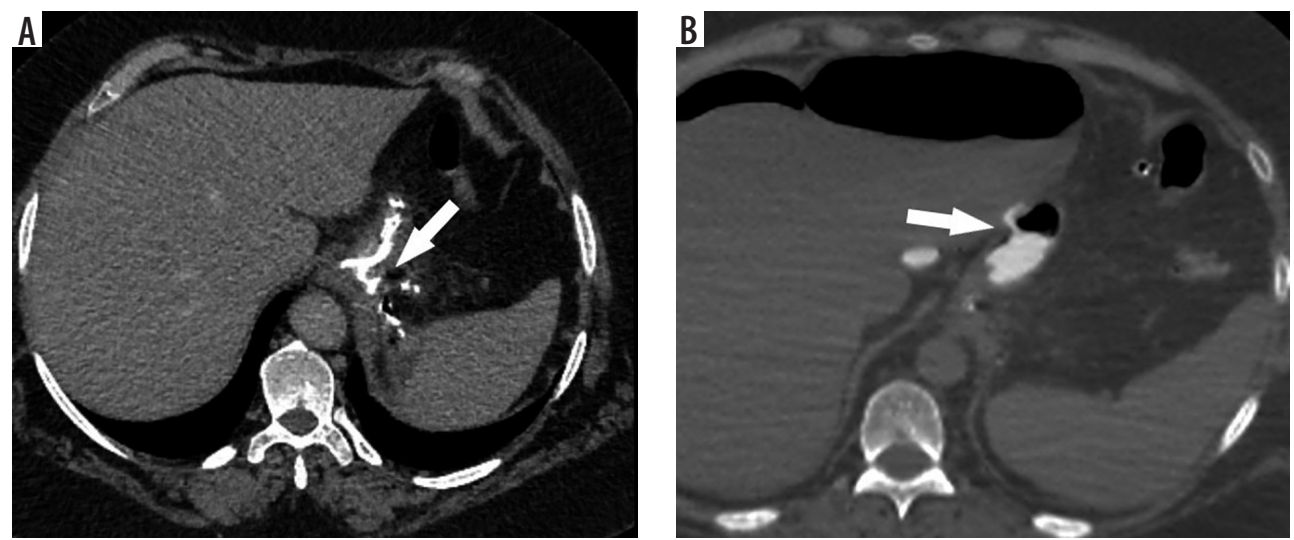

Figure 4. Computed tomography images showing extraluminal contrast medium (arrows) - which indicate a leakage 
Table 3. Computed tomography (CT) classification of leak after laparoscopic sleeve gastrectomy (LSG) [63]

\begin{tabular}{|l|c|c|c|}
\hline \multicolumn{1}{|l|}{ Type } & Collection on CT & \multicolumn{2}{|c|}{ Leak visualization } \\
\hline I & $<5 \mathrm{~cm}$ in left upper quadrant & $\mathrm{a}$ & No leak \\
\cline { 3 - 4 } & & $\mathrm{b}$ & Positive leak \\
\hline II & $>5 \mathrm{~cm}$ in left upper quadrant & $\mathrm{a}$ & No leak \\
& & $\mathrm{b}$ & Positive leak \\
\hline III & Diffuse abdominal collections & \multicolumn{2}{|}{} \\
\hline IV & Pleural (thoracic) collections & \multicolumn{1}{|}{} \\
\hline Type & Staple line localization \\
\hline S & Superior part of sleeve & \multicolumn{1}{|}{} \\
\hline M & \multicolumn{2}{|c}{ Middle } \\
\hline I & Inferior \\
\hline
\end{tabular}

pleural (thoracic) collections; the modifiers indicate the leak location in relation to the part of the gastric sleeve: $\mathrm{S}$ - superior, $\mathrm{M}$ - middle, I - inferior (Table 2). Nedelcu et al. created an algorithm for the management in different types of leak: type I leaks, which are clinically well tolerated, may be treated with starvation diet and intravenous antibiotics, and controlled with gastroscopy or CT scan. When conservative treatment is not effective enough or when any other type of leak is diagnosed, laparoscopic drainage should be performed; in type IV pleural drainage is necessary [63]. In persistent gastric leaks, treatment is usually tailored individually to each patient. Laparoscopic reoperation such as re-sleeve gastrectomy, conversion to Roux-en-Y gastric bypass, or total gastrectomy may be performed. Endoscopic management strategies include clipping, stenting, endoluminal vacuum or J-tube placement $[64,65]$ (Figure 5).

\section{Bleeding}

Postoperative bleeding usually occurs at the level of the gastric staple line, most often within the first 72 hours after surgery, and can be intra- or extraluminal. Intraluminal bleeding usually presents with haematemesis or melena stools. Signs of extraluminal bleeding include drops in serum haemoglobin level, tachycardia, and hypotension. Besides of the staple line, common origins of extraluminal bleeding are injuries to the spleen, liver, or abdominal wall at trocar entry sites $[66,67]$. In a majority of cases, conservative treatment is sufficient. Persistent active bleeding is an indication for surgical intervention or intravascular treatment depending on local expertise [68]. CT angiography is the method of choice for the detection of active bleeding; however, haemodynamically unstable patients should undergo surgical or intravascular intervention as soon as possible.

\section{Infection}

Infection is a possible early complication after LSG. Abscesses usually result from a leak and are most often located near the staple line in the left upper quadrant, most frequently in the subphrenic space [36]. Typical clinical manifestations include fever, abdominal pain, nausea, and vomiting. If there is a clinical suspicion of an infection, a CT scan of the abdomen should be performed. Portal venous phase offers the best means of detection of pathological fluid collections. Therapeutic drainage of the abscess can be conducted under CT guidance [34].

\section{Splenic infarction}

There is a wide range of anatomic variants of the arterial supply of the spleen. Anastomotic connections between the blood supply of the stomach and the spleen are often present $[69,70]$. During the LSG procedure, mobilization of the gastric fundus along with ligation of the short gastric vessels is necessary, which may cause insufficient splenic perfusion, most often in the upper pole of the spleen. The infarction may be asymptomatic or manifest like any other possible complication after LSG (fever, abdominal pain, tachycardia with increased inflammatory markers) and can lead to splenic abscess formation [71]. The diagnosis is established by conducting an angio-CT study of the abdomen (Figure 6). Gas within the infarcted area suggests abscess formation. In uncomplicated cases, conservative treatment is usually sufficient.
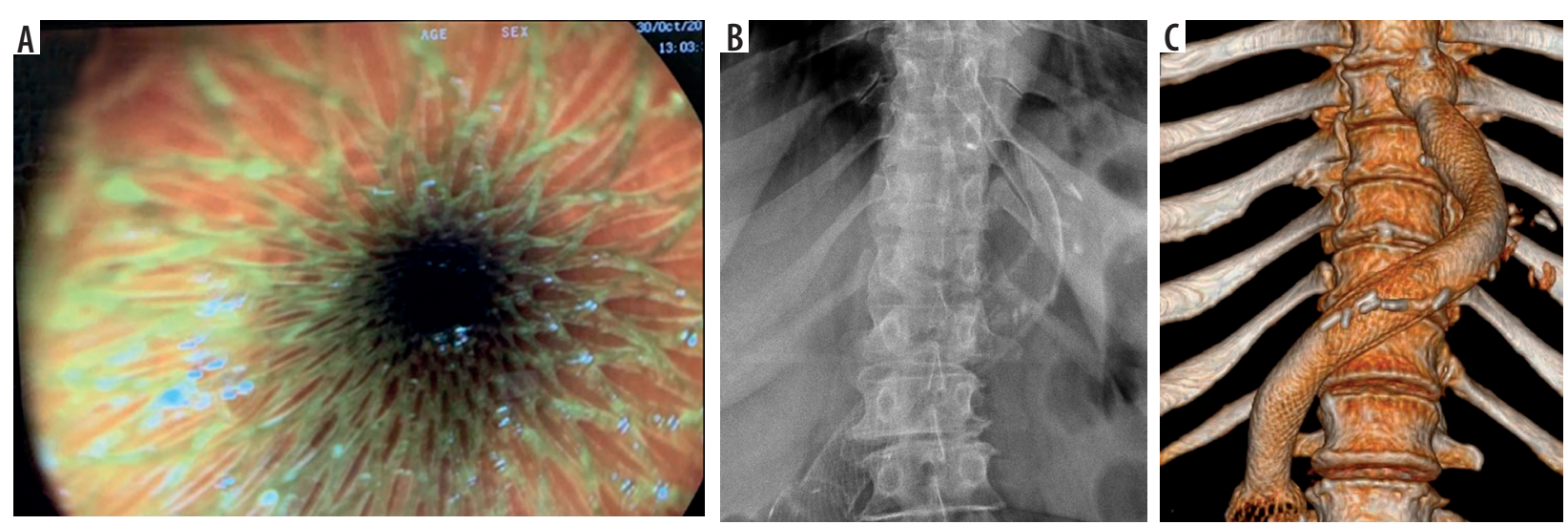

Figure 5. Gastroesophageal stent. A) endoscopy, B) radiography, C) computed tomography, 3D reconstruction. The stent should be placed from the lower part of esophagus to the proximal part of duodenum 


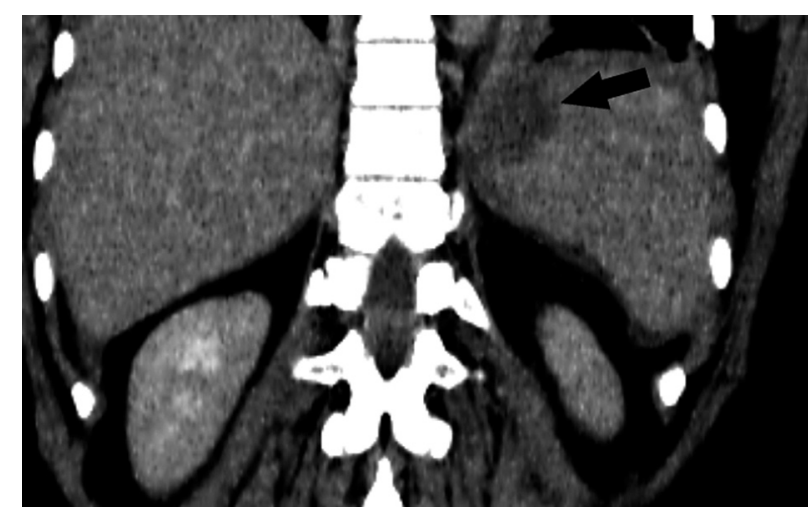

Figure 6. Computed tomography image showing a wedge-shaped, hypodense, non-enhancing region in the upper pole of a spleen (arrow)

\section{Stricture}

Another potential complication following LSG is gastric sleeve stricture. Clinical manifestations consist of dysphagia, stomach pain, nausea, and vomiting. The most vulnerable part of the stomach is the area of incisura angularis $[36,72]$. In the early postoperative period stenosis is usually caused by oedema and in a majority of cases is reversible. Stenosis may develop when the sleeve is too narrow, most commonly due to a technical error during the operation. Strictures can also be a side effect of oversewing of the staple line or could develop from fibrosis situated on the site of a previous leak, fistula, or haematoma [19,34] (Figure 7). Due to its dynamic character, UGI series is more effective in stricture detection than CT. The passage of swallowed contrast medium could be delayed or stopped [16]. In addition, gastric dilatation proximal to the stenotic segment is usually observed. When the stricture is at the level of gastroesophageal junction, the lower part of the oesophagus will be dilated [37]. The treatment of strictures, especially in the early postoperative period, is usually started in a conservative manner with proton-pump inhibitors and nasogastric tube placement or by endoscopic balloon dilatation [21,37]. Insertion of a stent is sometimes required $[16,36]$. In some cases surgical management, such as seromyotomy or conversion to RYGB (Roux-en-Y gastric bypass), is necessary [73].

Torsion of the gastric sleeve is a dangerous complication that can present similarly to stricture. It occurs when the anterior and the posterior wall of the stomach are not of identical length from the lesser curvature [21]. UGI shows dilatation of the proximal part of the gastric sleeve and delayed or stopped passage of the contrast medium, while the staple line exhibits a twisted course [19]. The management of this complication is with endoscopic or surgical intervention.

\section{Gastroesophageal reflux disease}

Gastroesophageal reflux disease (GERD) is a prevalent condition in morbidly obese patients. LSG can cause ex-

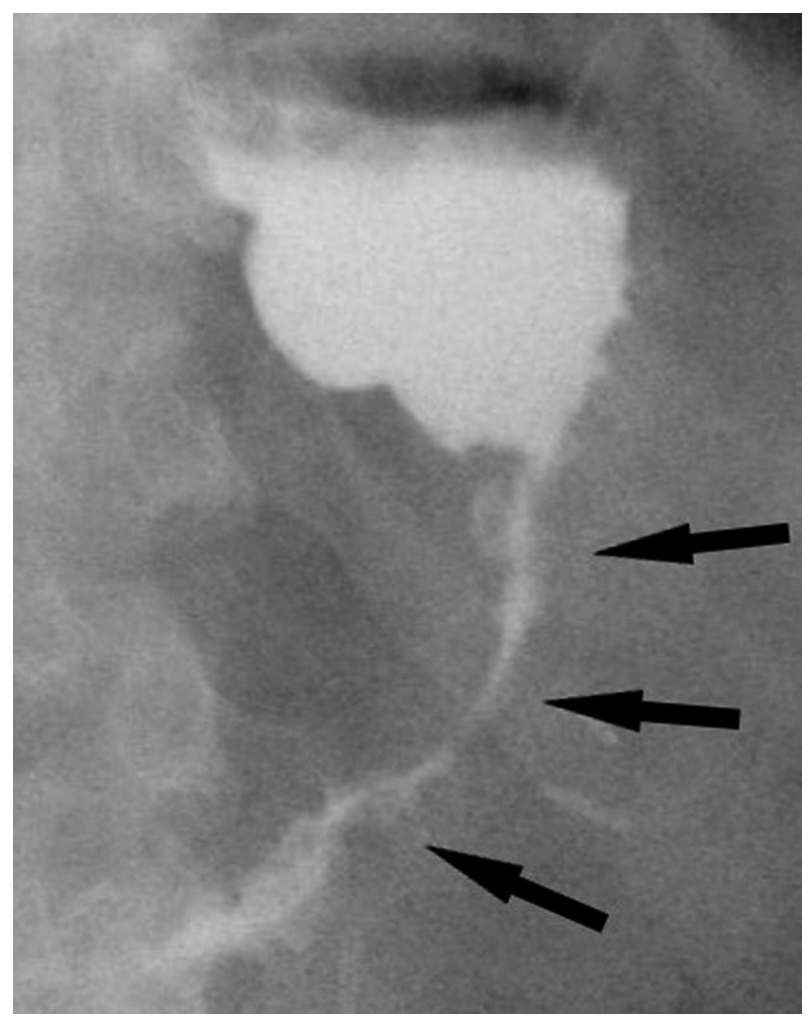

Figure 7. Upper gastrointestinal study - stricture after laparoscopic sleeve gastrectomy

acerbation, de novo appearance or reduction of its symptoms. Most of the studies suggest surgical technique and anatomical changes of the stomach as potential cause of the appearance of gastroesophageal reflux symptoms after LSG. Alleged reasons of GERD symptoms are reduction of lower oesophageal sphincter pressure, probably due to postoperative alteration of the angle of His, and enlargement or development of hiatal hernia $[36,74,75]$. Another reason could be gastric remnant stenosis near incisura angularis as a result of increased gastric fundal pressure [72]. As mentioned before, a superior pouch shape of gastric sleeve predisposes to gastroesophageal reflux $[16,37,38]$.

\section{Dilatation}

Gastric sleeve dilatation is a late complication occurring usually 2-3 years after the surgery. Gastric remnants have a tendency to extend progressively, which may lead to weight regain and may be a reason to perform revisional bariatric surgery [72,74]. A wide sleeve formation during a primary surgery is a predisposing factor for later dilatation. Other contributing factors include excessive pressure generated from consuming large meal volumes, repeated vomiting, or distal stricture of the stomach remnant [76]. Increase of gastric remnant volume and loss of its tubular shape may be observed on the UGI series [56] (Figure 8).

To sum up, UGI and CT are fundamental imaging techniques after LSG. For a long time, in multiple surgical centres, UGI was performed after every operation, 


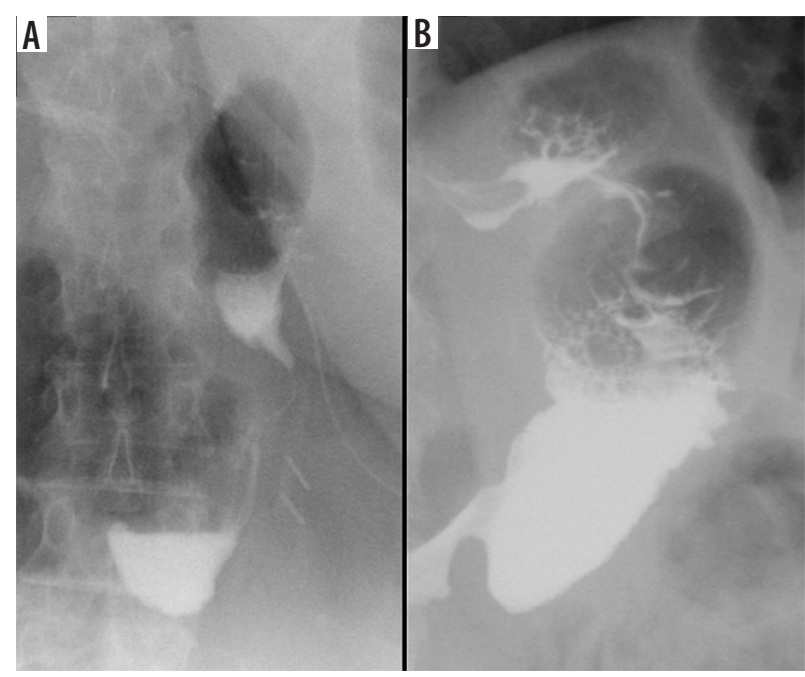

Figure 8. Upper gastrointestinal study. A) Early postoperative examination. B) Stomach dilatation 2 years after the surgery

but following numerous studies and controversies, UGI is no longer considered to be a routine examination $[23,57,59,60]$. According to the ERABS protocol, a liquid diet should be initiated without conducting UGI examination only a few hours after LSG [77]. UGI's low sensitivity in leak detection and high cumulative financial cost are perceived as significant drawbacks [57-60]. CT has a high sensitivity in leak detection and additionally visualizes other complications invisible on radiography, such as abscesses, active bleeding, or splenic infarction. UGI is still useful when functional disturbances are suspected, being very effective in the demonstration of strictures, dilatations, and abnormal passage. Gastric remnant volume calculation, performed either with UGI series or CT, is usually employed in scientific research.

\section{Conclusions}

There is no consensus on routine imaging after LSG in leak detection. Nowadays a lot of bariatric centres implement the ERABS protocol, which excludes routine use of radiological examinations in the postoperative period. If a patient experiences worrisome symptoms after the operation, CT is a method of choice in complication detection in the early postoperative period.

\section{Conflict of interest}

The authors report no conflict of interest.

\section{References}

1. Mitchell NS, Catenacci VA, Wyatt HR, et al. Obesity: overview of an epidemic. Psychiatr Clin North Am 2011; 34: 717-732.

2. Hurt RT, Kulisek C, Buchanan LA, et al. The obesity epidemic: challenges, health initiatives, and implications for gastroenterologists. Gastroenterol Hepatol (N Y) 2010; 6: 780-792.

3. Wang Y, Beydoun MA, Liang L, et al. Will all Americans become overweight or obese? estimating the progression and cost of the US obesity epidemic. Obesity (Silver Spring) 2008; 16: 2323-2330.

4. Kelly T, Yang W, Chen CS, et al. Global burden of obesity in 2005 and projections to 2030. Int J Obes (Lond) 2008; 32: 1431-1437.

5. http://appswhoint/gho/data/viewmain; 2013.

6. Kulaga Z, Gurzkowska B, Grajda A, et al. The prevalence of overweight and obesity among Polish pre-school-aged children. Dev Period Med 2016; 20: 143-149.

7. Kulaga Z, Grajda A, Gurzkowska B, et al. The prevalence of overweight and obesity among Polish school- aged children and adolescents. Przegl Epidemiol 2016; 70: 641-651.

8. Lagerros YT, Rossner S. Obesity management: what brings success? Therap Adv Gastroenterol 2013; 6: 77-88.

9. Wyatt HR. Update on treatment strategies for obesity. J Clin Endocrinol Metab 2013; 98: 1299-1306.

10. Mingrone G, Panunzi S, De Gaetano A, et al. Bariatric surgery versus conventional medical therapy for type 2 diabetes. N Engl J Med 2012; 366: 1577-1585.

11. Ozsoy Z, Demir E. Which bariatric procedure is the most popular in the world? A bibliometric comparison. Obes Surg 2018; 28: 23392352 .
12. Welbourn R, Hollyman M, Kinsman R, et al. Bariatric surgery worldwide: baseline demographic description and one-year outcomes from the fourth IFSO global registry report 2018. Obes Surg 2019; 29: 782-795.

13. English WJ, DeMaria EJ, Brethauer SA, et al. American Society for Metabolic and Bariatric Surgery estimation of metabolic and bariatric procedures performed in the United States in 2016. Surg Obes Relat Dis 2018; 14: 259-263.

14. Trastulli S, Desiderio J, Guarino S, et al. Laparoscopic sleeve gastrectomy compared with other bariatric surgical procedures: a systematic review of randomized trials. Surg Obes Relat Dis 2013; 9: 816-829.

15. Gartner D, Ernst A, Fedtke K, et al. Routine fluoroscopic investigations after primary bariatric surgery. Chirurg 2016; 87: 241-246.

16. Meredith I, Lim YK, Wickremesekera SK, et al. Experience of imaging following laparoscopic sleeve gastrectomy. ANZ J Surg 2016; 86: 568-571.

17. Budzyński A, Major P, Głuszek S, et al. Polskie rekomendacje w zakresie chirurgii bariatrycznej i metabolicznej. Medycyna Praktyczna - Chirurgia 2016; 6: 13-25.

18. Wang H, Lu J, Feng J, et al. Staple line oversewing during laparoscopic sleeve gastrectomy. Ann R Coll Surg Engl 2017; 99: 509-514.

19. Ekinci T, Stein MW, Mazzariol FS, et al. Laparoscopic sleeve gastrectomy: everything the radiologist needs to know. Clin Imaging 2017; 43: $36-41$.

20. Lazoura O, Zacharoulis D, Triantafyllidis G, et al. Symptoms of gastroesophageal reflux following laparoscopic sleeve gastrectomy are related to the final shape of the sleeve as depicted by radiology. Obes Surg 2011; 21: 295-299. 
21. Barnard SA, Rahman H, Foliaki A. The postoperative radiological features of laparoscopic sleeve gastrectomy. J Med Imaging Radiat Oncol 2012; 56: 425-431.

22. Millitz K, Moote DJ, Sparrow RK, et al. Pneumoperitoneum after laparoscopic cholecystectomy: frequency and duration as seen on upright chest radiographs. AJR Am J Roentgenol 1994; 163: 837-839.

23. Mittermair R, Sucher R, Perathoner A, et al. Routine upper gastrointestinal swallow studies after laparoscopic sleeve gastrectomy are unnecessary. Am J Surg 2014; 207: 897-901.

24. Lemanowicz A, Serafin Z. Imaging of patients treated with bariatric surgery. Pol J Radiol 2014; 79: 12-19.

25. Le NT, Robinson J, Lewis SJ. Obese patients and radiography literature: what do we know about a big issue? J Med Radiat Sci 2015; 62: 132-141.

26. Delko T, Mattiello D, Koestler T, et al. Computed tomography as primary postoperative follow-up after laparoscopic Roux-en-Y gastric bypass. World J Radiol 2018; 10: 1-6.

27. Deguines JB, Verhaeghe P, Yzet T, et al. Is the residual gastric volume after laparoscopic sleeve gastrectomy an objective criterion for adapting the treatment strategy after failure? Surg Obes Relat Dis 2013; 9: 660666.

28. Blanchet MC, Mesmann C, Yanes M, et al. 3D gastric computed tomography as a new imaging in patients with failure or complication after bariatric surgery. Obes Surg 2010; 20: 1727-1733.

29. Emara D, Sabry S, Abu El Enien M, Alexandria EG. Study of relationship between residual gastric volume andb weight loss after laparoscopic sleeve gastrectomy. ECR 2017. Poster number: C-0198. doi: 10.1594/ ecr2017/C-0198

30. Pawanindra L, Vindal A, Midha M, et al. Early post-operative weight loss after laparoscopic sleeve gastrectomy correlates with the volume of the excised stomach and not with that of the sleeve! Preliminary data from a multi-detector computed tomography-based study. Surg Endosc 2015; 29: 2921-2927.

31. Karcz WK, Kuesters S, Marjanovic G, et al. 3D-MSCT gastric pouch volumetry in bariatric surgery-preliminary clinical results. Obes Surg 2009; 19: 508-516.

32. Yazgan C, Balci S, Sahin T, Ozmen M. Imaging following minigastric bypass and sleeve gastrectomy: what every radiologists need to know. Poster on ECR 2016. Poster number: C-12642016. doi: 10.1594/ ecr2016/C-1264.

33. Hassan TA, Mohey N, Kamar WH. Clinical-radiologic evaluation of the complications of laparoscopic sleeve gastrectomy: value of multidetector CT. Egypt J Radiol Nucl Med 2015; 46: 879-884.

34. Chivot C, Robert B, Lafaye N, et al. Laparoscopic sleeve gastrectomy: imaging of normal anatomic features and postoperative gastrointestinal complications. Diagn Interv Imaging 2013; 94: 823-834.

35. Werquin C, Caudron J, Mezghani J, et al. Early imaging features after sleeve gastrectomy. J Radiol 2008; 89 (11 Pt 1): 1721-1728.

36. Ni Mhuircheartaigh J, Abedin S, Bennett AE, et al. Imaging features of bariatric surgery and its complications. Semin Ultrasound CT MR 2013; 34: 311-324.

37. Triantafyllidis G, Lazoura O, Sioka E, et al. Anatomy and complications following laparoscopic sleeve gastrectomy: radiological evaluation and imaging pitfalls. Obes Surg 2011; 21: 473-478.

38. Toro JP, Lin E, Patel AD, et al. Association of radiographic morphology with early gastroesophageal reflux disease and satiety control after sleeve gastrectomy. J Am Coll Surg 2014; 219: 430-438.
39. Garcia-Diaz JJ, Ferrer-Marquez M, Moreno-Serrano A, et al. Outcomes, controversies and gastric volume after laparoscopic sleeve gastrectomy in the treatment of obesity. Cir Cir 2016; 84: 369-375.

40. Ferrer-Marquez M, Garcia-Diaz JJ, Moreno-Serrano A, et al. Changes in gastric volume and their implications for weight loss after laparoscopic sleeve gastrectomy. Obes Surg 2017; 27: 303-309.

41. Vidal P, Ramon JM, Busto M, et al. Residual gastric volume estimated with a new radiological volumetric model: relationship with weight loss after laparoscopic sleeve gastrectomy. Obes Surg 2014; 24 : 359-363.

42. Pomerri F, Foletto M, Allegro G, et al. Laparoscopic sleeve gastrectomy--radiological assessment of fundus size and sleeve voiding. Obes Surg 2011; 21: 858-863.

43. Weisstein EW. Ellipsoid. From MathWorld - A Wolfram Web Resource. http://mathworldwolframcom/Ellipsoidhtml.

44. Braghetto I, Cortes C, Herquinigo D, et al. Evaluation of the radiological gastric capacity and evolution of the BMI 2-3 years after sleeve gastrectomy. Obes Surg 2009; 19: 1262-1269.

45. Salman MAA EM, Ragab AA, Hegazy TO. Relationship between the resected stomach volume and early postoperative weight loss following laparoscopic sleeve gastrectomy. Int Surg J 2018; 5: 1180-1185.

46. Kueper MA, Kramer KM, Kirschniak A, et al. Laparoscopic sleeve gastrectomy: standardized technique of a potential stand-alone bariatric procedure in morbidly obese patients. World J Surg 2008; 32: 1462-1465.

47. Goitein D, Goitein O, Feigin A, et al. Sleeve gastrectomy: radiologic patterns after surgery. Surg Endosc 2009; 23: 1559-1563.

48. Goitein D, Zendel A, Westrich G, et al. Postoperative swallow study as a predictor of intermediate weight loss after sleeve gastrectomy. Obes Surg 2013; 23: 222-225.

49. Sakran N, Goitein D, Raziel A, et al. Gastric leaks after sleeve gastrectomy: a multicenter experience with 2,834 patients. Surg Endosc 2013; 27: 240-245.

50. Iossa A, Abdelgawad M, Watkins BM, et al. Leaks after laparoscopic sleeve gastrectomy: overview of pathogenesis and risk factors. Langenbecks Arch Surg 2016; 401: 757-766.

51. Gagner M, Buchwald JN. Comparison of laparoscopic sleeve gastrectomy leak rates in four staple-line reinforcement options: a systematic review. Surg Obes Relat Dis 2014; 10: 713-723.

52. Burgos AM, Braghetto I, Csendes A, et al. Gastric leak after laparoscopic-sleeve gastrectomy for obesity. Obes Surg 2009; 19: 16721677.

53. Csendes A, Burdiles P, Burgos AM, et al. Conservative management of anastomotic leaks after 557 open gastric bypasses. Obes Surg 2005; 15: 1252-1256.

54. Csendes A, Diaz JC, Burdiles P, et al. Classification and treatment of anastomotic leakage after extended total gastrectomy in gastric carcinoma. Hepatogastroenterology 1990; 37 Suppl 2: 174-177.

55. Chivot C, Rebibo L, Robert B, et al. Value of routine upper gastrointestinal swallow study after laparoscopic sleeve gastrectomy. Surg Obes Relat Dis 2017; 13: 758-765.

56. Levine MS, Carucci LR. Imaging of bariatric surgery: normal anatomy and postoperative complications. Radiology 2014; 270: 327-341.

57. Wahby M, Salama AF, Elezaby AF, et al. Is routine postoperative gastrografin study needed after laparoscopic sleeve gastrectomy? Experience of 712 cases. Obes Surg 2013; 23: 1711-1717. 
58. Terterov D, Leung PH, Twells LK, et al. The usefulness and costs of routine contrast studies after laparoscopic sleeve gastrectomy for detecting staple line leaks. Can J Surg 2017; 60: 335-341.

59. Mizrahi I, Tabak A, Grinbaum R, et al. The utility of routine postoperative upper gastrointestinal swallow studies following laparoscopic sleeve gastrectomy. Obes Surg 2014; 24: 1415-1419.

60. Brockmeyer JR, Simon TE, Jacob RK, et al. Upper gastrointestinal swallow study following bariatric surgery: institutional review and review of the literature. Obes Surg 2012; 22: 1039-1043.

61. de Aretxabala X, Leon J, Wiedmaier G, et al. Gastric leak after sleeve gastrectomy: analysis of its management. Obes Surg 2011; 21: 1232-1237.

62. Alharbi SR. Computed tomography-based diagnosis of post-laparoscopic sleeve gastrectomy gastric leak. J Clin Imaging Sci 2020; 10: 8 .

63. Nedelcu M, Skalli M, Delhom E, et al. New CT scan classification of leak after sleeve gastrectomy. Obes Surg 2013; 23: 1341-1343.

64. Leeds SG, Burdick JS. Management of gastric leaks after sleeve gastrectomy with endoluminal vacuum (E-Vac) therapy. Surg Obes Relat Dis 2016; 12: 1278-1285.

65. Gagner M, Hutchinson C, Rosenthal R. Fifth International Consensus Conference: current status of sleeve gastrectomy. Surg Obes Relat Dis 2016; 12: 750-756.

66. Sarkhosh K, Birch DW, Sharma A, et al. Complications associated with laparoscopic sleeve gastrectomy for morbid obesity: a surgeon's guide. Can J Surg 2013; 56: 347-352.

67. Herron D, Roohipour R. Complications of Roux-en-Y gastric bypass and sleeve gastrectomy. Abdom Imaging 2012; 37: 712-718.

68. Yong S, Poh B, Eng A, et al. Post-operative bleeding complications in laparoscopic sleeve gastrectomy: sources, solutions and lessons learnt from a single cohort of patients. Dig Med Res 2019; 2: 6.

69. Michalik M, Budziński R, Orłowski M, et al. Splenic infarction as a complication of laparoscopic sleeve gastrectomy. Videosurgery and Other Miniinvasive Techniques 2011; 6: 92-98.

70. Stamou KM, Menenakos E, Gomatos IP, et al. Clinical implications of sleeve gastrectomy as a source of spleen infarction or ischemia. Obes Surg 2011; 21: 1490-1493.

71. Nassour F, Schoucair NM, Tranchart H, et al. Delayed intra splenic abscess: a specific complication following laparoscopic sleeve gastrectomy. Obes Surg 2018; 28: 589-593.

72. Katz DP, Lee SR, Nachiappan AC, et al. Laparoscopic sleeve gastrectomy: a guide to postoperative anatomy and complications. Abdom Imaging 2011; 36: 363-371.

73. Rosenthal RJ; International Sleeve Gastrectomy Expert Panel. International Sleeve Gastrectomy Expert Panel Consensus Statement: best practice guidelines based on experience of $>12,000$ cases. Surg Obes Relat Dis 2012; 8: 8-19.

74. Shah S, Shah V, Ahmed AR, et al. Imaging in bariatric surgery: service set-up, post-operative anatomy and complications. Br J Radiol 2011; 84: 101-111.

75. Melissas J, Braghetto I, Molina JC, et al. Gastroesophageal reflux disease and sleeve gastrectomy. Obes Surg 2015; 25: 2430-2435.

76. Gagner M, Rogula T. Laparoscopic reoperative sleeve gastrectomy for poor weight loss after biliopancreatic diversion with duodenal switch. Obes Surg 2003; 13: 649-654.

77. Awad S, Carter S, Purkayastha S, et al. Enhanced recovery after bariatric surgery (ERABS): clinical outcomes from a tertiary referral bariatric centre. Obes Surg 2014; 24: 753-758. 